The Annals of Applied Probability

2002, Vol. 12, No. 4, 1227-1246

\title{
OPTIMAL INVESTMENT WITH TRANSACTION COSTS AND WITHOUT SEMIMARTINGALES
}

\author{
By PAOLO GUASONI
}

\section{Università di Pisa}

\begin{abstract}
We consider a general class of optimization problems in financial markets with incomplete information and transaction costs. Under a noarbitrage condition strictly weaker than the existence of a martingale measure, and when asset prices are quasi-left-continuous processes, we show the existence of optimal strategies.

Applications include maximization of expected utility, minimization of coherent risk measures and hedging of contingent claims.
\end{abstract}

1. Introduction. One of the oldest results in Mathematical Finance, due to Kreps and Yan [16, 20] (but see also Delbaen and Schachermayer [4] for a more general version), states that in a frictionless market where free lunches are not allowed, all assets must be semimartingales. Since the gain of a trading strategy is given by its integral with respect to the asset process, this result legitimates the use of the heavy machinery of stochastic integration, with all its far-reaching consequences.

In presence of proportional transaction costs, semimartingales are not the only arbitrage-free assets anymore. At the same time, not all strategies are permitted (or meaningful), as trading volume must remain finite. In other words, as the class of reasonable integrators enlarges, the set of admissible integrands shrinks, and it turns out that integration can still be defined consistently. Indeed, this can be done in an elementary way, path by path. Of course, all the powerful results on semimartingales and stochastic integration cannot be applied to this setting, but the General Theory of Processes still provides a lot of information on these integrals.

We consider a market with one riskless asset (used as numeraire) and $d$ risky assets. In this market, an economic agent is endowed with some initial capital $c$, faces a liability (e.g., a contingent claim to hedge) $H$ at time $T$, and trades in the available assets to optimize some objective function $\rho$ (which can be an expected utility, a risk measure, etc.). For each unit of numeraire (e.g., euro) traded into or out of the $i$ th risky asset, the agent is charged a fee of $k_{t}^{i}$ units. One can also think of this setting as a model convention under which the reference asset price $X_{t}^{i}$ is chosen so that the bid and ask prices are respectively $\left(1-k_{t}^{i}\right) X_{t}^{i}$ and $\left(1+k_{t}^{i}\right) X_{t}^{i}$. This optimization problem can be written as

$$
\min _{\theta \in \mathcal{A}_{c}^{k}} \rho\left(V_{T}^{c}(\theta)-H\right)
$$

Received September 2001; revised March 2002.

AMS 2000 subject classifications. 60H30, 62P05, 91B30, 26A45.

Key words and phrases. Transaction costs, incomplete markets, coherent risk measures, utility maximization. 
where $\mathcal{A}_{c}^{k}$ is the set of admissible strategies and $V_{T}^{c}$ is the liquidation value of the agent portfolio at time $T$.

The above settings provide a convenient framework for different problems, such as utility maximization and hedging of contingent claims. In the frictionless case, the utility maximization problem goes back to Merton [17] and has been studied by a number of different authors, among whom we mention Karatzas, Lehoczky, Shreve and $\mathrm{Xu}$ [13], the first to solve this problem in an incomplete market setting, and Kramkov and Schachermayer [15], who provide a necessary and sufficient condition for the existence of solutions in the semimartingale case.

More work in this area stems from the problem of hedging contingent claims in incomplete markets, where utility maximization is replaced by risk minimization. For instance, Cvitanić [1] has studied the shortfall minimization problem for incomplete frictionless markets, while Cvitanić and Karatzas [2] have considered the problem of hedging in presence of transaction costs.

In the main result of this paper, we show the existence of optimal strategies when asset prices are quasi-left-continuous, under a suitable no-arbitrage condition. Of course, this condition is strictly weaker than the existence of an equivalent martingale measure, which would force us to the semimartingale case.

As in [8], the existence of solutions is obtained with a direct method technique, finding a convergence where optimizing sequences are relatively compact, and the risk functional is lower semicontinuous. In particular, we exploit the additional compactness introduced by transaction costs in the set of admissible strategies, which is stable under small perturbations of asset processes, and hence does not depend on the semimartingale property (as opposed to [8], where compactness results for classes of integrable martingales are used).

The quasi-left-continuity assumption is required only in the proof of the semicontinuity of the risk functional. For continuous processes, this easily follows from duality arguments, which hold path by path regardless of the probabilistic context of the problem. On the contrary, the extension to the quasileft-continuous case requires some extra effort, as we show that the functional is lower semicontinuous almost surely, relying on the representation of jumps for cadlag processes.

The paper is organized as follows: in Section 2 we describe the model of a financial market with frictions, and show how integration can be defined consistently. Section 3 contains the compactness theorem for admissible strategies, which holds under a no-arbitrage condition. In Section 4 we show that the existence of optimal strategies follows from the semicontinuity of the portfolio value. We show the case of continuous processes separately, as the proofs are considerably simpler. In Section 5 we discuss how the utility maximization problem can be embedded in this framework, and show with a counterexample that the compactness result may not hold for some degenerate but still arbitragefree markets. 
2. The market model. We consider a standard model for a financial market, with a filtered probability space $\left(\Omega, \mathcal{F},\left(\mathcal{F}_{t}\right)_{0 \leq t \leq T}, P\right)$, where the filtration $\mathcal{F}_{t}$ satisfies the usual assumptions. In this market there is a riskless asset, used as numeraire, and hence assumed identically equal to 1 .

We have $d$ risky assets, given by an $\mathbb{R}^{d}$-valued process $X=\left(\left(X_{t}^{i}\right)_{i=1}^{d}\right)_{t \in[0, T]}$, adapted to the filtration $\mathcal{F}_{t}$. Transaction costs are proportional, and we denote by $k=\left(\left(k_{t}^{i}\right)_{i=1}^{d}\right)_{t \in[0, T]}$ the cost for each unit transacted (hence the cost for a one-share transaction on the $i$ th asset at time $t$ is $k_{t}^{i} X_{t}^{i}$ ).

For vector-valued processes, we shall use the upper index to denote the component, and the usual lower index to denote time. When we consider sequences of processes, we shall use the upper index to denote the position in the sequence, using the lower position for both the component and time.

In general, we can expect $k$ to depend both on $t$ and $\omega$, reflecting changing liquidity conditions at different times and circumstances. Hence, we will allow $k$ to be an adapted, strictly positive stochastic process, discussing further assumptions when needed.

In frictionless markets, a self-financed strategy is uniquely defined by the number of shares $\theta=\left(\left(\theta_{t}^{i}\right)_{i=1}^{d}\right)_{t \in[0, T]}$ held in each risky asset at each time. Therefore, starting from an initial capital $c$, the portfolio value at time $t$ is given by

$$
c+\int_{(0, t]} \theta_{s} d X_{S}
$$

with the integral representing the gain up to time $t$. In the presence of transaction costs, in general we have two processes $L=\left(\left(L_{t}^{i}\right)_{i=1}^{d}\right)_{t \in[0, T]}$ and $M=$ $\left(\left(M_{t}^{i}\right)_{i=1}^{d}\right)_{t \in[0, T]}$ representing respectively the cumulative number of shares purchased and sold up to time $t$, with the obvious relation $\theta_{t}=L_{t}-M_{t}$. Hence the liquidation value of a portfolio at time $t$ can be written as

$$
V_{t}^{c}(\theta)=c+\int_{(0, t]} \theta_{s} d X_{s}-\sum_{i=1}^{d}\left(\int_{[0, t]} k_{s}^{i} X_{s}^{i} d\left(L^{i}+M^{i}\right)_{s}+k_{t}^{i}\left|\theta_{t}^{i}\right| X_{t}^{i}\right),
$$

where the last two terms represent respectively the cost of the trading strategy and the final cost of liquidation. This expression can be further simplified, under the additional (financially sound) assumption that purchases and sales should not overlap, to avoid dissipation of wealth.

To translate this requirement in mathematical terms, we need some notation. Given a function of bounded variation $\theta$, we denote by $D \theta$ its derivative in the sense of distributions, by $|D \theta|$ the total variation measure associated with $D \theta$ and by $|D \theta|_{t}=|D \theta|[0, t]$. These definitions trivially extend componentwise to vector-valued strategies $\theta=\left(\left(\theta_{t}^{i}\right)_{i=1}^{d}\right)_{t \in[0, T]}$ as $D \theta=\left(\left(D \theta_{t}^{i}\right)_{i=1}^{d}\right)_{t \in[0, T]},|D \theta|=$ 
$\left(\left(|D \theta|_{t}^{i}\right)_{i=1}^{d}\right)_{t \in[0, T]}$. Denote also $\|D \theta\|_{t}=\sum_{i=1}^{d}\left|D \theta^{i}\right|_{t}$. The no-dissipation condition above then translates into $L_{t}+M_{t}=|D \theta|_{t}$, and we have that

$$
V_{t}^{c}(\theta)=c+\int_{(0, t]} \theta_{s} d X_{s}-\sum_{i=1}^{d}\left(\int_{[0, t]} k_{s}^{i} X_{s}^{i} d\left|D \theta^{i}\right|_{s}+k_{t}^{i}\left|\theta_{t}^{i}\right| X_{t}^{i}\right) .
$$

With an abuse of notation, we identify the vector $k$ with the $d \times d$ diagonal matrix with elements $\left(k^{1}, \ldots, k^{d}\right)$ so that we can rewrite the above expression as

$$
V_{t}^{c}(\theta)=c+\int_{(0, t]} \theta_{S} d X_{s}-\int_{[0, t]} k_{s} X_{S} \cdot d|D \theta|_{s}-k_{t} X_{t} \cdot\left|\theta_{t}\right| .
$$

REMARK 2.1. In our model, only transactions between risky assets and cash are permitted, and all transaction costs are charged to the cash account. Hence, this choice is appropriate whenever these features are present, most notably in stock markets, but also in bond and commodity markets. By contrast, in foreign exchange markets all assets can be indifferently exchanged, and in this case the approach of Kabanov et al. [10, 12, 11] should rather be employed.

Now it remains to give a meaning to the gain process $\int_{[0, t]} \theta_{s} d X_{s}$, since we consider asset processes which are not necessarily semimartingales. The Bichteler-Dellacherie theorem characterizes semimartingales as the largest class of integrators for general predictable strategies, therefore we will have to restrict the class of integrands. In fact, in our setting of a market with frictions, we already consider only strategies of finite variation, and stochastic integrals for this class can be defined path by path.

Definition 2.2. Let $\theta: \mathbb{R}^{+} \mapsto \mathbb{R}$ be a left-continuous function of finite variation, and $X$ a cadlag function. Then we define the integral of $\theta$ with respect to $X$ as (Dellacherie and Meyer [6], 8.1)

$$
\int_{(0, t]} \theta_{s} d X_{s}=\theta_{t^{+}} X_{t}-\theta_{0^{+}} X_{0}-\int_{(0, t]} X_{s} d D \theta_{s^{+}}
$$

For a general function $\theta$ of finite variation, we define:

$$
\int_{(0, t]} \theta_{s} d X_{s}=\int_{(0, t]} \theta_{s^{-}} d X_{s}+\sum_{\substack{s \leq t \\ \theta_{s}-\neq \theta_{s}}}\left(\theta_{s}-\theta_{s^{-}}\right) \Delta X_{s},
$$

where the first term in the right is defined by (2). To denote these integrals we shall indifferently use the notation $\int_{(0, t]} \theta_{S} d X_{S}$ or $(\theta \cdot X)_{t}$.

REMARK 2.3. The integral defined above trivially extends path by path to finite variation processes $\theta=\left(\left(\theta_{t}^{i}\right)_{i=1}^{d}\right)_{t \in[0, T]}$ and cadlag processes $X=$ $\left(\left(X_{t}^{i}\right)_{i=1}^{d}\right)_{t \in[0, T]}$. In addition, when $X$ is a semimartingale, the integral in (2) coincides with the usual stochastic integral (see Dellacherie and Meyer [6], 8.1). 
The definition in (3) is given for completeness, since for predictable $\theta$ it provides the financially natural extension of the gain process. However, we shall soon show that in our setting of quasi-left-continuous $X$ we may simply consider left continuous $\theta$, since the extra term in (3) disappears.

To verify the good definition of (3) one should check that the series in the righthand side converges. Indeed, since $\theta$ has bounded variation we have that (recall that $X_{t}^{*}=\sup _{s \leq t}\left|X_{s}\right|$ )

$$
\sum_{\substack{s \leq t \\ \theta_{s^{-}} \neq \theta_{s}}}\left(\theta_{s}-\theta_{s^{-}}\right) \Delta X_{s} \leq 2 X_{t}^{*} \sum_{s \leq t}\left|\theta_{s}-\theta_{s^{-}}\right| \leq 2 X_{t}^{*}|D \theta|_{t}
$$

as needed. Finally, we stick to the usual convention that $\theta_{0^{-}}=0$, so that (2) can be written as

$$
\int_{(0, t]} \theta_{s} d X_{s}=\theta_{t^{+}} \cdot X_{t}-\int_{[0, t]} X_{S} \cdot d D \theta_{s^{+}}
$$

In this paper we consider asset processes $X$ with the following properties:

ASSUMPTION 2.4. We assume the following:

(i) $X$ is a cadlag process, adapted to the filtration $\mathcal{F}_{t}$;

(ii) $X_{t}>0$ a.s. for all $t \in[0, T]$;

(iii) $X$ is quasi-left-continuous; that is, $X_{\tau}=X_{\tau^{-}}$a.s. for all predictable stopping times $\tau$.

The next proposition shows that for these processes we only need to consider left-continuous strategies.

PROPOSITION 2.5. Let $X$ be a quasi-left-continuous process, and $\theta$ a predictable, finite variation process. Then we have that:

$$
\int_{[0, t]} \theta_{s} d X_{s}=\int_{[0, t]} \theta_{s^{-}} d X_{s} \quad \text { a.s. }
$$

PROOF. Since $X$ is a cadlag adapted process, $\{\Delta X \neq 0\}=\bigcup_{k} \llbracket \tau_{k} \rrbracket$, where $\tau_{k}$ is a sequence of stopping times with disjoint graphs, and each of them is either predictable or totally inaccessible (see, e.g., Dellacherie and Meyer [5]). When $X$ is quasi-left-continuous, up to a null set we can assume that all $\tau_{k}$ are totally inaccessible. It follows that

$$
\int_{[0, t]} \theta_{s} d X_{s}-\int_{[0, t]} \theta_{s^{-}} d X_{s}=\sum_{\tau_{k}}\left(\theta_{\tau_{k}}-\theta_{\tau_{k}^{-}}\right) \cdot \Delta X_{\tau_{k}}
$$

Since $\theta$ is a predictable process of finite variation, we can define

$$
J_{t}=\sum_{s \leq t}\left(\theta_{s}-\theta_{s^{-}}\right)
$$


which is a predictable, cadlag process. Hence it follows (see Dellacherie and Meyer [5], Chapter IV, Theorem 88B) that the set $\left\{(t, \omega): \theta_{t}-\theta_{t^{-}} \neq 0\right\}=$ $\{\Delta J \neq 0\}=\bigcup_{k} \llbracket \sigma_{k} \rrbracket$ where $\sigma_{k}$ is a sequence of predictable stopping times.

However, this means that $\theta_{\tau_{k}}-\theta_{\tau_{k}^{-}}$is indistinguishable from the null process for all $k$, and the proof is complete.

In the context of transaction costs, we can easily generalize the definition of admissible strategy as follows:

DEFINITION 2.6. A predictable finite variation process $\theta$ is called $c$-admissible if for all $t$ we have that $V_{t}^{c}(\theta) \geq 0$ a.e.

We denote by $\mathcal{A}_{c}^{k}$ the class of $c$-admissible strategies with transaction costs $k$.

Accordingly we give a definition of arbitrage strategy.

DEFINITION 2.7. An admissible strategy $\theta$ is an arbitrage opportunity if, for some $t, V_{t}^{0}(\theta) \geq 0, P\left(V_{t}^{0}(\theta)>0\right)>0$.

REMARK 2.8. Kabanov and Stricker [12] distinguish two kinds of arbitrage in the context of transaction costs. While a strong arbitrage offers the usual opportunity of a riskless profit, a weak arbitrage merely allows one to build a position in the risky asset, recovering all initial transaction costs. The above definition corresponds to strong arbitrage, as it is given in terms of the liquidation value $V_{t}^{0}$.

With transaction costs, the absence of arbitrage generally does not imply the existence of a (local) martingale measure for $X$. In addition, since the semimartingale property is preserved under a change to an equivalent measure, such condition would force us to the semimartingale case.

In our setting the natural substitute for a martingale measure would be a condition equivalent to no arbitrage in the presence of transaction costs, but in continuous time this still seems an open problem, at least to the author's knowledge.

However, we can consider the following sufficient condition, which allows for processes that are not semimartingales:

DEFINITION 2.9. Given an adapted, strictly positive process $\gamma=$ $\left(\left(\gamma_{t}^{i}\right)_{i=1}^{d}\right)_{t \in[0, T]}$, a process $X$ is $\gamma$-arbitrage free if there exists a process $\tilde{X}$ and a probability $Q$ equivalent to $P$ such that $\left(1-\gamma_{t}^{i}\right) X_{t}^{i} \leq \tilde{X}_{t}^{i} \leq\left(1+\gamma_{t}^{i}\right) X_{t}^{i}$ almost surely for all $t, i$ and $\tilde{X}$ is a local martingale under $Q$.

We recall the following no-arbitrage criterion (see [7]): 
PROPOSITION 2.10. If $X$ is $\gamma$-arbitrage free, and $\gamma \leq k$ (i.e., $\gamma_{t}^{i} \leq k_{t}^{i}$ a.s. for all $t, i)$, then $X$ is arbitrage-free with transaction costs $k$.

In fact, our results will depend on the following slightly stronger condition:

DEFINITION 2.11. We say that a market with transaction costs $k$ is compact arbitrage free (CAF) if it is $\gamma$-arbitrage free and

$$
\min _{1 \leq i \leq d} \underset{t \in[0, T]}{\operatorname{essinf}}\left(k_{t}^{i}-\gamma_{t}^{i}\right)>0 \quad \text { a.s. }
$$

REMARK 2.12. Note that the $\gamma$-arbitrage free condition is trivially satisfied with $\gamma=0$ if there exists a martingale measure for $X$.

In the case of finite $\Omega$ the converse of Proposition 2.10 holds true (see Jouini and Kallal [9] and Kabanov and Stricker [12]).

Very recently, Schachermayer [18] (see also Kabanov, Rasonyi and Stricker [11] for an alternative proof) has shown that in discrete time with a general $\Omega$, the analogous of martingale measures with transaction costs are strictly consistent price systems, and their existence is equivalent to the robust no-arbitrage property, which guarantees compactness properties in $L^{0}$ very similar to those established in this paper.

3. Compactness of admissible strategies. In this section we show that transaction costs, coupled with the admissibility and no-arbitrage conditions, generate a strong compactness property for sequences of admissible strategies.

Roughly speaking, the idea is that any sequence of strategies should be uniformly bounded in trading volume, otherwise the admissibility condition would be violated. Such an estimate on volume will translate into one for the number of shares at each instant, and compactness will follow.

Of course, the problem is to understand in which sense such boundedness holds, and what kind of compactness it implies. One can reason as follows: we aim at results independent of agent's preferences and views or, in mathematical terms, that are invariant under a change to an equivalent probability. As a result, we cannot rely on integrability properties of asset prices, which depend on the particular probability chosen by the agent.

On the contrary, boundedness in probability (or in $L^{0}$ ) has the desired property. This is easily seen by the following lemma, which must be well known, but since we have no reference we report it along with its short proof.

LEMMA 3.1. Let $X$ and $\left\{Y_{i}\right\}_{i \in I}$ be strictly positive, finite-valued, random variables. Then $\left\{Y_{i}\right\}_{i \in I}$ is bounded in $L^{0}$ if and only if $\left\{X Y_{i}\right\}_{i \in I}$ is bounded in $L^{0}$. 
Proof. Suppose that $\left\{Y_{i}\right\}_{i \in I}$ is bounded in $L^{0}$. We obviously have

$$
\begin{aligned}
P\left(X Y_{i}>M\right) & =P\left(X Y_{i}>M, X \leq N\right)+P\left(X Y_{i}>M, X>N\right) \\
& \leq P\left(Y_{i}>\frac{M}{N}\right)+P(X>N) .
\end{aligned}
$$

With a suitable choice of $M$ and $N$, both these terms are arbitrarily small, as $X$ is finite valued, and $\left\{Y_{i}\right\}_{i \in I}$ is bounded in $L^{0}$. The reverse implication follows from the first one, denoting $X^{\prime}=\frac{1}{X}$ and $Y_{i}^{\prime}=X Y_{i}$.

We now give the compactness result: note that it generally does not hold in the frictionless case. In this sense, the existence of solutions to optimization problems with transaction costs may actually be easier than their frictionless counterparts, as admissibility becomes a more binding condition.

Proposition 3.2. Let $X$ satisfy Assumption 2.4 and the (CAF) condition (Definition 2.11). If $\theta^{n} \in \mathcal{A}_{c}^{k}$ is a sequence of finite variation processes, then there exists a sequence $\eta^{n} \in \operatorname{conv}\left(\theta^{n}, \theta^{n+1}, \ldots\right)$ such that $\eta^{n}$ converges a.s. in $d t d P(\omega)$ to a finite variation process $\theta \in \mathcal{A}_{c}^{k}$.

We break the proof of Proposition 3.2 into three parts. First we recall the following lemma from Delbaen and Schachermayer [4]:

LeMma 3.3 ([4], Lemma A1.1). Let $\left(f_{n}\right)_{n>1}$ be a sequence of $[0, \infty)$ valued measurable functions on a probability space $(\Omega, \mathcal{F}, P)$. There exists a sequence $g_{n} \in \operatorname{conv}\left(f_{n}, f_{n+1}, \ldots\right)$ such that $\left(g_{n}\right)_{n \geq 1}$ converges almost surely to a $[0, \infty]$ valued function $g$.

If $\operatorname{conv}\left(f_{n}, f_{n+1}, \ldots\right)$ is bounded in $L^{0}$, then $g$ is finite almost surely. If there are $\alpha>0$ and $\delta>0$ such that for all $n: P\left(f_{n}>\alpha\right)>\delta$, then $P(g>0)>0$.

The next lemma can be seen as a compactness result for Fatou convergence (see Kramkov [14], Lemma 4.2) but here convergence is sought within the class of predictable processes (see also Kabanov and Last [10], Lemma 3.4 for a similar result in the context of transaction costs):

LEMMA 3.4. Let $\theta^{n}$ be a sequence of processes of finite variation, such that the set $\operatorname{conv}\left(\left\{\left\|D \theta^{n}\right\|_{T}\right\}_{n \in \mathbb{N}}\right)$ is bounded in $L^{0}(\Omega)$.

Then there is a sequence $\eta^{n} \in \operatorname{conv}\left(\theta^{n}, \theta^{n+1}, \ldots\right)$ such that $\eta^{n}$ converges a.s. in $d t d P(\omega)$ to a finite variation process $\theta$.

PROOF. By the Hahn decomposition, a function of bounded variation is a difference of two monotone functions. Hence we can write $\theta_{t}^{n}=L_{t}^{n}-M_{t}^{n}$, where $L^{n}$ and $M^{n}$ are increasing processes which are essentially unique under the condition that $\left|D \theta^{n}\right|_{t}=L_{t}^{n}+M_{t}^{n}$. 
Given a convex combination $\left(\alpha_{k}\right)_{k=n}^{\infty}$, we have that:

$$
\sum_{k \geq n} \alpha_{k} L_{t}^{k} \leq \sum_{k \geq n} \alpha_{k}\left|D \theta^{k}\right|_{t} \leq \sum_{k \geq n} \alpha_{k}\left|D \theta^{k}\right|_{T} \quad \text { a.e. }
$$

therefore $\operatorname{conv}\left(L_{t}^{n}, L_{t}^{n+1}, \ldots\right)$ is bounded in $L^{0}$. By Lemma 3.3, up to a sequence of convex combinations we can assume that $L_{t}^{n}$ converges almost surely to some $\mathbb{R}^{d}$-valued variable $L_{t}$.

By a diagonalization argument, up to a sequence of convex combinations we can assume that, for all $t \in \mathscr{D}=\{0, T\} \cup(\mathbb{Q} \cap(0, T)), L_{t}^{n}$ converges almost surely to a process $\left(L_{t}\right)_{t \in \mathscr{D}}$. Clearly, $L_{t}$ is an increasing process.

We define $\tilde{L}_{t}=\sup _{s \in \mathbb{Q} \cap(0, t)} L_{s}$. Since $\tilde{L}$ is left-continuous, it is obviously predictable. We now show that, for each $\omega, L_{t}^{n} \rightarrow \tilde{L}_{t}$ everywhere but in the discontinuity points of $\tilde{L}(\omega)$, which are at most a countable set. In fact, if $L(\omega)$ is continuous in $t$, for any $\varepsilon>0$ we can find $p, q \in \mathscr{D}$ such that $p<t<q$ and $L_{p}(\omega) \leq L_{q}(\omega) \leq L_{p}(\omega)+\varepsilon$. Passing to the limit, we get

$$
L_{p}(\omega) \leq \liminf _{n \rightarrow \infty} L_{t}^{n}(\omega) \leq \limsup _{n \rightarrow \infty} L_{t}^{n}(\omega) \leq L_{p}(\omega)+\varepsilon .
$$

Since $\varepsilon$ is arbitrary, it follows that $L_{t}^{n}$ converges to $\tilde{L}_{t}$.

Repeating the same argument for $M$, we obtain that, up to a sequence of convex combinations, $L^{n}$ and $M^{n}$ respectively converge a.s. in $d t d P(\omega)$ to increasing processes $\tilde{L}$ and $\tilde{M}$. As a result, $L_{t}^{n}-M_{t}^{n}$ converges to $\theta_{t}=\tilde{L}_{t}-\tilde{M}_{t}$, which is a finite variation process.

REMARK 3.5. Note that the proof of Lemma 3.4 provides a stronger result than the one stated. In fact, is shows that for almost every $\omega$ there exists a countable set $N(\omega) \subset[0, T]$ such that $\theta^{n}(\omega) \rightarrow \theta(\omega)$ for all $t \notin N(\omega)$.

So far no reference to the asset process is present. The assumptions on $X$ in Proposition 3.2 will now be needed to link the admissibility of trading strategies to the boundedness condition in Lemma 3.4.

Proof of Proposition 3.2. For any $\theta \in \mathcal{A}_{c}^{k}$, we have

$$
\begin{aligned}
-c \leq & (\theta \cdot X)_{T}-\int_{[0, T]} k_{S} X_{S} \cdot d|D \theta|_{s}-k_{T} X_{T} \cdot\left|\theta_{T}\right| \\
= & (\theta \cdot X)_{T}-\left(\int_{[0, T]} \gamma_{s} X_{S} \cdot d|D \theta|_{s}+\gamma_{T} X_{T} \cdot\left|\theta_{T}\right|\right) \\
& -\left(\int_{[0, T]}\left(k_{S}-\gamma_{S}\right) X_{S} \cdot d|D \theta|_{s}+\left(k_{T}-\gamma_{T}\right) X_{T} \cdot\left|\theta_{T}\right|\right) .
\end{aligned}
$$

Now, we claim that

$$
(\theta \cdot X)_{T}-\left(\int_{[0, T]} \gamma_{S} X_{S} \cdot d|D \theta|_{S}+\gamma_{T} X_{T} \cdot\left|\theta_{T}\right|\right) \leq(\theta \cdot \tilde{X})_{T}
$$


where $\tilde{X}$ satisfies the properties in Definition 2.9. In fact, recall that $X$ is $\gamma$ arbitrage free, and denote by $\varepsilon_{t}=\tilde{X}_{t}-X_{t}$. By assumption we have that $\left|\varepsilon_{t}^{i}\right| \leq$ $\gamma_{t}^{i} X_{t}$ and integrating by parts, we obtain

$$
\begin{aligned}
(\theta \cdot \tilde{X})_{T} & =(\theta \cdot(X+\varepsilon))_{T}=(\theta \cdot X)_{T}+\theta_{T} \cdot \varepsilon_{T}-\theta_{0^{+}} \cdot \varepsilon_{0}-\int_{(0, T]} \varepsilon_{S} \cdot d D \theta_{S} \\
& =(\theta \cdot X)_{T}+\theta_{T} \cdot \varepsilon_{T}-\int_{[0, T]} \varepsilon_{S} \cdot d D \theta_{S} \\
& \geq(\theta \cdot X)_{T}-\int_{[0, T]} \gamma_{S} X_{S} \cdot d|D \theta|_{S}-\gamma_{T} X_{T} \cdot\left|\theta_{T}\right|
\end{aligned}
$$

which proves the claim. From (5) and (6) it follows that

$$
-c \leq(\theta \cdot \tilde{X})_{T}-\delta_{T}\left(\xi_{T} \cdot|D \theta|_{T}+X_{T} \cdot\left|\theta_{T}\right|\right),
$$

where $\delta_{t}=\min _{1 \leq i \leq d} \operatorname{essinf}_{s \in[0, t]}\left(k_{s}^{i}-\gamma_{s}^{i}\right)$ and $\xi_{t}=\inf _{s \in[0, t]} X_{s}$. Under the measure $Q$, the stochastic integral $(\theta \cdot \tilde{X})_{T}$ is a local martingale bounded from below, thus a supermartingale. Taking expectations, we have

$$
E\left[\delta_{T} \xi_{T} \cdot|D \theta|_{T}+\delta_{T} X_{T} \cdot\left|\theta_{T}\right|\right] \leq c .
$$

This inequality is clearly stable under convex combinations of strategies, and therefore the closed convex hull of the set $\left\{\delta_{T} \xi_{T} \cdot|D \theta|_{T}: \theta \in \mathcal{A}_{c}^{k}\right\}$ is bounded in $L^{1}(Q)$ and hence in $L^{0}(Q)$.

By the (CAF) assumption, $\delta_{T}>0$ a.s. Also, note that $\xi_{T}>0$ a.e.: to see this, denote by $\tau=\inf \left\{t: X_{t}=0\right.$ or $\left.X_{t^{-}}=0\right\}$. Since $X$ is $\gamma$-arbitrage free, we have that $\tau=\inf \left\{t: \tilde{X}_{t}=0\right.$ or $\left.\tilde{X}_{t^{-}}=0\right\}$. However, then we obtain that $\tau>T$ a.s., as $\tilde{X}$ is a strictly positive martingale under $Q$.

This implies that we can apply Lemma 3.1, and we obtain that the closed convex hull of $\left\{\|D \theta\|_{T}: \theta \in \mathcal{A}_{c}^{k}\right\}$ is bounded in $L^{0}(P)$.

Now, let $\theta^{n} \in \mathcal{A}_{c}^{k}$ be a sequence of finite variation processes. From the above discussion it follows that the assumptions of Lemma 3.4 are satisfied; therefore we can assume, up to a sequence of convex combinations, that $\theta^{n}$ converges a.s. in $d t d P(\omega)$ to some finite variation process $\theta$. The admissibility of $\theta$ will follow from Proposition 4.7 (or Proposition 4.4, if $X$ is continuous).

REMARK 3.6. The (CAF) condition in Lemma 3.4 cannot be relaxed to allow for $\gamma^{i}=k^{i}$ for some $i$. Indeed, in the last section we show with a counterexample that in this case, which represents the edge of the no-arbitrage condition, the above compactness properties may break down. Of course, this does not exclude the existence of solutions, but requires arguments different from those shown here.

4. Existence of optimal strategies. We start by defining the class of risk functionals used in this paper. 
DEFINITION 4.1. A lower semicontinuous convex decreasing functional is a function $\rho: L^{0} \mapsto \mathbb{R} \cup\{+\infty\}$, with the following properties:

(i) $\rho$ is convex;

(ii) if $X \leq Y$ almost surely, then $\rho(X) \geq \rho(Y)$;

(iii) $\rho$ has the Fatou property. If $X_{n} \rightarrow X$ a.e., and $X_{n} \geq-a$ a.e. for some $a>0$, then

$$
\rho(X) \leq \liminf _{n \rightarrow \infty} \rho\left(X_{n}\right)
$$

REMARK 4.2. Definition 4.1 is a natural modification of the analogous definition in [8] to the present setting, where no integrability conditions are present. In particular, here the Fatou property is only required for sequences uniformly bounded from below. This means that the above definition includes all $\sigma$-additive coherent risk measures as defined by Delbaen [3].

The rest of this section deals with the lower semicontinuity of the above functionals with respect to a.s. convergence in $d t d P(\omega)$. For these functionals, it will be enough to check that the portfolio value $V_{t}^{c}$ is upper semicontinuous, and here we shall need the quasi left-continuity of $X$.

We begin with a lemma which links the pointwise convergence of $\theta_{t}^{n}(\omega)$ to the weak star convergence of the measures $D \theta^{n}(\omega)$.

LEMMA 4.3. If $\operatorname{conv}\left(\left\{\left\|D \theta^{n}\right\|_{T}\right\}_{n \in \mathbb{N}}\right)$ is bounded in $L^{0}$ and $\theta_{t}^{n} \rightarrow \theta_{t}$ a.s. in $d t d P(\omega)$, then up to a sequence of convex combinations $D \theta_{t}^{n} \rightarrow D \theta_{t}$ for a.e. $\omega$.

PROOF. By Lemma 3.3, up to a sequence of convex combinations we can assume that $\lim _{n \rightarrow \infty}\left\|D \theta^{n}\right\|_{T}=V(\omega)$, with $V<\infty$ a.e.

We have

$$
\left\{\limsup _{n \rightarrow \infty}\left\|D \theta^{n}\right\|_{T}>M\right\}=\left\{\liminf _{n \rightarrow \infty}\left\|D \theta^{n}\right\|_{T}>M\right\}=\bigcup_{k} \bigcap_{n \geq k}\left\{\left\|D \theta^{n}\right\|_{T}>M\right\}
$$

and hence, since $\left\|D \theta^{n}\right\|_{T}$ is bounded in $L^{0}$,

$$
\begin{aligned}
& P\left(\limsup _{n \rightarrow \infty}\left\|D \theta^{n}\right\|_{T}>M\right) \\
& \quad=P\left(\bigcup_{k} \bigcap_{n \geq k}\left\{\left\|D \theta^{n}\right\|_{T}>M\right\}\right) \leq \sup _{n} P\left(\left\|D \theta^{n}\right\|_{T}>M\right) \leq \varepsilon .
\end{aligned}
$$

It follows that $\lim \sup _{n \rightarrow \infty}\left\|D \theta^{n}\right\|_{T}<\infty$ a.e. and hence $\sup _{n}\left\|D \theta^{n}\right\|_{T}<\infty$ a.e. Since $\theta_{t}^{n} \rightarrow \theta_{t}$ a.e., the thesis follows by the Lebesgue dominated convergence theorem. 
4.1. The case of continuous processes. Here we consider the case where $X$ is a continuous process. In this setting, the semicontinuity of $V_{t}^{c}$ can be shown using only arguments of duality between continuous functions and Radon measures.

Proposition 4.4. If $\theta^{n} \in \mathcal{A}_{c}^{k}, \operatorname{conv}\left(\left\{\left\|D \theta^{n}\right\|_{T}\right\}_{n \in \mathbb{N}}\right)$ is bounded in $L^{0}$ and $\theta_{t}^{n} \rightarrow \theta_{t}$ a.s. in $d t d P(\omega)$, then we have

$$
V_{T}^{c}(\theta) \geq \limsup _{n \rightarrow \infty} V_{T}^{c}\left(\theta^{n}\right) \quad \text { for a.e. } \omega .
$$

Proof. By Lemma 4.3, we have that $D \theta_{t}^{n} \rightarrow D \theta_{t}$, and hence, integrating by parts,

$$
\begin{aligned}
(\theta \cdot X)_{T} & =X_{T} \cdot \theta_{T}-\int_{[0, T]} X_{t} \cdot d D \theta_{t} \\
& =\lim _{n \rightarrow \infty}\left(X_{T} \cdot \theta_{T}^{n}-\int_{[0, T]} X_{t} \cdot d D \theta_{t}^{n}\right)=\lim _{n \rightarrow \infty}\left(\theta^{n} \cdot X\right)_{T}
\end{aligned}
$$

and, by the semicontinuity of the variation, we have

which completes the proof.

$$
\int_{[0, T]} X_{t} \cdot d|D \theta|_{t} \leq \liminf _{n \rightarrow \infty} \int_{[0, T]} X_{t} \cdot d\left|D \theta^{n}\right|_{t},
$$

From the upper semicontinuity of $V_{T}^{c}$ we easily obtain the lower semicontinuity of our functional:

LEMMA 4.5. Let $\rho$ be a functional as in Definition 4.1, $H$ an $\mathcal{F}_{T}$-measurable random variable and $c>0$. Denoting by $F: \theta \mapsto \rho\left(V_{T}^{c}(\theta)-H\right)$, we have:

(i) $F$ is convex;

(ii) $F$ is lower semicontinuous with respect to $d t d P(\omega)$-a.s. convergence.

PROOF. Follows by a convexity argument and by Fatou's lemma, exactly as in [8], lemma 4.3.

The existence result is then an easy corollary:

Proposition 4.6. Let $X$ be a continuous process satisfying Assumption 2.4 and the (CAF) condition. If $\rho$ has the properties in Definition 4.1, then the problem

$$
\min _{\theta \in \mathcal{A}_{c}^{k}} \rho\left(V_{T}^{c}(\theta)-H\right)
$$

admits a solution.

Proof. Let $\theta^{n} \in \mathcal{A}_{c}^{k}$ be a minimizing sequence. From Proposition 3.2 we obtain a sequence $\eta^{n} \in \operatorname{conv}\left(\theta^{n}, \theta^{n+1}, \ldots\right)$ such that $\eta^{n} \rightarrow \theta \in \mathcal{A}_{c}^{k}$ a.s. in $d t d P(\omega)$. By the semicontinuity of $\rho$ (Lemma 4.5), it follows that $\theta$ is a minimizer. 
4.2. The quasi-left-continuous case. We now come to the more general case where $X$ is a quasi-left-continuous process on the whole interval $[0, T]$. Note that this assumption implies the continuity of $X$ at $T$.

In this setting, arguments of duality between continuous functions and signed measures cannot be applied directly to show that the portfolio value is lower semicontinuous, as the discontinuities of $X$ may be relevant for the limit measure $D \theta$. A simple example is given by a limit strategy which changes immediately after a jump has occurred.

Nevertheless, if the jumps of $X$ are totally inaccessible, the cases where convergence fails are negligible, as admissible strategies must be predictable. This is the main idea of the next proof, though somewhat hidden in the inevitable technical details.

PROPOSITION 4.7. If $\theta^{n} \in \mathcal{A}_{c}^{k}, \theta_{t}^{n} \rightarrow \theta_{t}$ a.s. in $d t d P(\omega)$ and $\operatorname{conv}\left(\left\{\left\|D \theta^{n}\right\|_{T}\right\}_{n \in \mathbb{N}}\right)$ is bounded in $L^{0}$, then up to a subsequence

$$
V_{T}^{c}(\theta) \geq \limsup _{n \rightarrow \infty} V_{T}^{c}\left(\theta^{n}\right) \quad \text { for a.e. } \omega .
$$

We break the proof of Proposition 4.7 into two lemmas.

LEMMA 4.8. If $D \theta^{n} \rightarrow D \theta$ a.s., then we have

$$
\int_{[0, T]} X_{S} \cdot d|D \theta|_{s} \leq \liminf _{n \rightarrow \infty} \int_{[0, T]} X_{s} \cdot d\left|D \theta^{n}\right|_{s} \quad \text { for a.e. } \omega .
$$

In addition, if $\left\|D \theta^{n}\right\|_{T} \rightarrow\|D \theta\|_{T}$ a.s. (i.e., $D \theta^{n}$ converges to $D \theta$ in variation), then we obtain

$$
\int_{[0, T]} X_{s} \cdot d|D \theta|_{s}=\lim _{n \rightarrow \infty} \int_{[0, T]} X_{s} \cdot d\left|D \theta^{n}\right|_{s} \quad \text { for a.e. } \omega .
$$

PROOF. By a change of variable, we have

$$
\int_{[0, T]} X_{s} \cdot d|D \theta|_{s}=\int_{0}^{\infty}|D \theta|_{T}(X>x) d x
$$

therefore it is sufficient to check that

$$
|D \theta|(X>x) \leq \liminf _{n \rightarrow \infty}\left|D \theta^{n}\right|(X>x) .
$$

Of course, the problem here is that the set $\{X>x\}$ is not necessarily open, as $X$ may have discontinuities. However, $X$ has only totally inaccessible jumps, therefore $\{\Delta X \neq 0\}=\bigcup_{k} \llbracket \sigma_{k} \rrbracket$ a.s., where $\sigma_{k}$ is a sequence of totally inaccessible stopping times.

We denote by $\tau_{k}=\inf \left\{t \geq \sigma_{k}: X_{t} \leq x\right\}$, and define recursively

$$
\begin{aligned}
& \tilde{\sigma}_{1}=\sigma_{1}, \quad A_{1}=\llbracket \sigma_{1}, \tau_{1} \llbracket, \\
& \tilde{\sigma}_{k}=\left.\sigma_{k}\right|_{\left\{\sigma_{k} \notin A_{k-1}\right\}}, \quad A_{k}=A_{k-1} \cup \llbracket \sigma_{k}, \tau_{k} \llbracket,
\end{aligned}
$$


where $\left.\tau\right|_{A}=\tau 1_{A}+\infty 1_{\Omega \backslash A}$ for any stopping time $\tau$. It is easy to see that the set $\left\{\sigma_{k} \notin A_{k-1}\right\}$ is $\tilde{\mathcal{F}}_{\sigma_{k}}$-measurable, and hence $\tilde{\sigma}_{k}$ is a stopping time for all $k$.

We denote by

$$
B_{x}=\{X>x\} \backslash\left(\bigcup_{k \in \mathbb{N}} \llbracket \tilde{\sigma}_{k}, \tau_{k} \llbracket\right)
$$

$\tilde{\sigma}_{k}$ and $\alpha_{k}, \beta_{k}$ (we stick to the convention that $[a, b)$ is empty if $b \leq a$ ). and observe that $B_{x}$ is open a.s., as the process $X$ is continuous outside the random set $A_{\infty}=\bigcup_{k \geq 1} A_{k}$. It follows that:

$$
|D \theta|\left(B_{x}\right) \leq \liminf _{n \rightarrow \infty}\left|D \theta^{n}\right|\left(B_{x}\right) \quad \text { for a.e. } \omega
$$

so it suffices to show that the same property holds for the stochastic intervals $\llbracket \tilde{\sigma}_{k}, \tau_{k} \llbracket$.

Up to a subsequence, we can assume that $\left|D \theta^{n}\right| \rightarrow \mu$, where $\mu \geq|D \theta|$. We define the predictable process

$$
\delta_{t}=\liminf _{n \rightarrow \infty}\left|D \theta^{n}\right|(0, t)-\mu(0, t) .
$$

Since we have that

$$
\mu[0, t] \geq \limsup _{n \rightarrow \infty}\left|D \theta^{n}\right|[0, t] \geq \liminf _{n \rightarrow \infty}\left|D \theta^{n}\right|(0, t) \geq \mu(0, t)
$$

it follows that $0 \leq \delta_{t} \leq \mu\{t\}$ and hence $\delta_{t}>0$ for at most countably many $t$.

As a result (see [5], Chapter IV, Theorem 88), $\left\{(t, \omega): \delta_{t}>0\right\}=\bigcup_{k} \llbracket \pi_{k} \rrbracket$, where $\left\{\pi_{k}\right\}_{k \in \mathbb{N}}$ is a sequence of predictable stopping times. It follows that $P\left(\pi_{j}=\tilde{\sigma}_{k}\right)=0$ for all $j, k$, and hence $\lim _{n \rightarrow \infty}\left|D \theta^{n}\right|\left(\rrbracket 0, \tilde{\sigma}_{k} \mathbb{\amalg}\right)=\mu\left(\rrbracket 0, \tilde{\sigma}_{k} \mathbb{L}\right)$ a.s.

We have that

$$
\begin{aligned}
\mu\left(\rrbracket 0, \tau_{k} \llbracket\right) & \leq \liminf _{n \rightarrow \infty}\left|D \theta^{n}\right|\left(\rrbracket 0, \tau_{k} \llbracket\right) \\
& =\lim _{n \rightarrow \infty}\left|D \theta^{n}\right|\left(\rrbracket 0, \tilde{\sigma}_{k} \llbracket\right)+\liminf _{n \rightarrow \infty}\left|D \theta^{n}\right|\left(\llbracket \tilde{\sigma}_{k}, \tau_{k} \llbracket\right) \\
& =\mu\left(\rrbracket 0, \tilde{\sigma}_{k} \llbracket\right)+\liminf _{n \rightarrow \infty}\left|D \theta^{n}\right|\left(\llbracket \tilde{\sigma}_{k}, \tau_{k} \llbracket\right),
\end{aligned}
$$

whence

$$
\mu\left(\llbracket \tilde{\sigma}_{k}, \tau_{k} \llbracket\right) \leq \lim _{n \rightarrow \infty}\left|D \theta^{n}\right|\left(\llbracket \tilde{\sigma}_{k}, \tau_{k} \llbracket\right),
$$

which completes the proof of (9).

The proof of (10) is analogous, and will be omitted.

LEMMA 4.9. If $\theta_{t}^{n} \rightarrow \theta_{t}$ a.s. in $d t d P(\omega)$ and $\operatorname{conv}\left(\left\{\left\|D \theta^{n}\right\|_{T}\right\}_{n \in \mathbb{N}}\right)$ is bounded in $L^{0}$, then up to a subsequence $\left(\theta^{n} \cdot X\right)_{T}$ converges in probability to $(\theta \cdot X)_{T}$. 
ProOF. We have that

$$
\begin{aligned}
\left(\theta^{n} \cdot X\right)_{T} & =X_{T} \cdot \theta_{T}^{n}-\int_{[0, T]} X_{t} \cdot d D \theta_{t}^{n} \\
& =X_{T} \cdot \theta_{T}^{n}-\int_{[0, T]} X_{t} \cdot d\left(D \theta^{n}\right)_{t}^{+}-\int_{[0, T]} X_{t} \cdot d\left(D \theta^{n}\right)_{t}^{-},
\end{aligned}
$$

where $\left(D \theta^{n}\right)^{+}$and $\left(D \theta^{n}\right)^{-}$denote respectively the positive and negative parts in the Hahn decomposition of $D \theta^{n}$. Up to subsequences, we can assume that $\left|D \theta^{n}\right|_{T}$ converges a.s. and hence that $\left(D \theta^{n}\right)^{+} \rightarrow v^{+}$and $\left(D \theta^{n}\right)^{-} \rightarrow v^{-}$, where $v^{+}$and $v^{-}$ are positive vector measures. Applying Lemma 4.8 to the last two integrals above, we obtain that

$$
\begin{aligned}
\lim _{n \rightarrow \infty}\left(\theta^{n} \cdot X\right)_{T} & =X_{T} \cdot \theta_{T}-\int_{[0, T]} X_{t} \cdot d v_{t}^{+}-\int_{[0, T]} X_{t} d \cdot v_{t}^{-} \\
& =X_{T} \cdot \theta_{T}-\int_{[0, T]} X_{t} \cdot d D \theta_{t}=(\theta \cdot X)_{T} .
\end{aligned}
$$

Proof of Proposition 4.7. The thesis follows from Lemmas 4.3, 4.9 and 4.8 .

As in the continuous case, the existence of minimizers is easily obtained:

PROPOSITION 4.10. Let $X$ be a quasi-left-continuous process satisfying Assumption 2.4 and the (CAF) condition. If $\rho$ has the properties in Definition 4.1, then the problem

$$
\min _{\theta \in \mathcal{A}_{c}^{k}} \rho\left(V_{T}^{c}(\theta)-H\right)
$$

admits a solution.

Proof. As in Proposition 4.6, it follows from Proposition 3.2 and 4.7.

\section{Examples and counterexamples.}

5.1. Utility maximization. It is natural to embed the utility maximization problem in the framework of Proposition 4.6 by choosing $\rho(X)=E[-U(X)]$. However, while conditions (i) and (ii) in Definition 4.1 are trivially satisfied for any utility function $U$, the Fatou property (iii) generally does not hold, unless $U$ is bounded.

However, the existence of an optimal solution requires that only maximizing sequences satisfy the Fatou property. In fact, in the frictionless case, Kramkov and Schachermayer [15] have shown with convex duality arguments that a necessary and sufficient condition for existence is that $U$ has reasonable asymptotic elasticity, defined as follows: 
DEFINITION 5.1. The asymptotic elasticity of an increasing concave function $U$ is defined by

$$
A E_{+\infty}(U)=\limsup _{x \rightarrow \infty} \frac{x U^{\prime}(x)}{U(x)}
$$

and $U$ has reasonable asymptotic elasticity if $A E_{+\infty}(U)<1$.

Here we show that an alternative proof of this result, due to Schachermayer [19], can easily be adapted to the case of transaction costs.

THEOREM 5.2. Let $X$ be a quasi-left-continuous process satisfying Assumption 2.4 and the (CAF) condition. Let $U: \mathbb{R}^{+} \mapsto \mathbb{R}$ be an increasing concave function, such that $A E_{+\infty}(U)<1$.

If $\max _{\theta \in \mathcal{A}_{c}^{k}} E\left[U\left(V_{T}^{c}(\theta)\right)\right]<\infty$, then the problem

$$
\max _{\theta \in \mathcal{A}_{c}^{k}} E\left[U\left(V_{T}^{c}(\theta)\right)\right]
$$

admits a solution.

We need the following lemma of Schachermayer [19]:

LEMMA 5.3 [19]. Let $\left(f_{n}\right)_{n=1}^{\infty} \geq 0$ be random variables on $(\Omega, \mathcal{F}, P)$ converging a.s. to $f_{0}$. Suppose that $\lim _{n \rightarrow \infty} E\left[f_{n}\right]=E\left[f_{0}\right]+\alpha$, for some $\alpha>0$. Then for all $\varepsilon>0$ there exist $n, m>\varepsilon^{-1}$ and disjoint sets $A_{n}, A_{m}$ such that the following conditions are satisfied:

(i) $f_{n} \geq \varepsilon^{-1}$ on $A_{n}$ and $f_{m} \geq \varepsilon^{-1}$ on $A_{m}$;

(ii) $E\left[f_{n} 1_{A_{n}}\right]>\alpha-\varepsilon$ and $E\left[f_{m} 1_{A_{m}}\right]>\alpha-\varepsilon$;

(iii) $E\left[f_{n} 1_{\Omega \backslash\left(A_{n} \cup A_{m}\right)}\right]>E\left[f_{0}\right]-\varepsilon$ and $E\left[f_{m} 1_{\Omega \backslash\left(A_{n} \cup A_{m}\right)}\right]>E\left[f_{0}\right]-\varepsilon$.

Proof OF THEOREM 5.2. Let $\theta^{k}$ be a maximizing sequence for (14). Since $A E_{+\infty}(U)<1$, by Lemma 6.3 in [15] there exists some $\beta>1$ such that $U\left(\frac{x}{2}\right)>\frac{\beta}{2} U(x)$ for all $x \geq x_{0}$.

Since $X$ satisfies the (CAF) condition, by Proposition 3.2 we can assume up to a sequence of convex combinations that $\theta^{k} \rightarrow \theta \in \mathcal{A}_{c}^{k}$ a.s. in $d t d P$. By Proposition 4.7, we have that

$$
V_{T}^{c}(\theta) \geq \limsup _{k \rightarrow \infty} V_{T}^{c}\left(\theta^{k}\right) \quad \text { a.s. in } P .
$$

We need to show that $\lim _{k \rightarrow \infty} E\left[U\left(V_{T}^{c}\left(\theta^{k}\right)\right)\right] \leq E\left[U\left(V_{T}^{c}(\theta)\right)\right]$. By contradiction, suppose that, up to a subsequence:

$$
\lim _{n \rightarrow \infty} E\left[U\left(V_{T}^{c}\left(\theta^{n}\right)\right)\right]-E\left[U\left(V_{T}^{c}(\theta)\right)\right]=\alpha>0 .
$$


Then, by Lemma 5.3 we could find $n, m$ arbitrarily large and $A_{n}, A_{m}$ such that

$$
\begin{aligned}
E\left[\left(\frac{V_{T}^{c}\left(\theta^{n}\right)+V_{T}^{c}\left(\theta^{m}\right)}{2}\right)\right]= & E\left[U\left(\frac{V_{T}^{c}\left(\theta^{n}\right)+V_{T}^{c}\left(\theta^{m}\right)}{2}\right) 1_{\Omega \backslash\left(A_{n} \cup A_{m}\right)}\right] \\
& +E\left[U\left(\frac{V_{T}^{c}\left(\theta^{n}\right)+V_{T}^{c}\left(\theta^{m}\right)}{2}\right) 1_{A_{n} \cup A_{m}}\right] .
\end{aligned}
$$

By the condition $A E_{+\infty}(U)<1$, for the second term in the right we have

$$
\begin{gathered}
E\left[U\left(\frac{V_{T}^{c}\left(\theta^{n}\right)+V_{T}^{c}\left(\theta^{m}\right)}{2}\right) 1_{A_{n} \cup A_{m}}\right] \geq \beta E\left[\frac{U\left(V_{T}^{c}\left(\theta^{n}\right)+V_{T}^{c}\left(\theta^{m}\right)\right)}{2} 1_{A_{n} \cup A_{m}}\right] \\
\geq \frac{\beta}{2}\left(E\left[U\left(V_{T}^{c}\left(\theta^{n}\right)\right) 1_{A_{n}}\right]+E\left[U\left(V_{T}^{c}\left(\theta^{m}\right)\right) 1_{A_{m}}\right]\right) \geq \beta(\alpha-\varepsilon)
\end{gathered}
$$

while for the first term

$$
\begin{aligned}
& E\left[U\left(\frac{V_{T}^{c}\left(\theta^{n}\right)+V_{T}^{c}\left(\theta^{m}\right)}{2}\right) 1_{\Omega \backslash\left(A_{n} \cup A_{m}\right)}\right] \\
& \quad \geq \frac{1}{2}\left(E\left[U\left(V_{T}^{c}\left(\theta^{n}\right)\right) 1_{\Omega \backslash\left(A_{n} \cup A_{m}\right)}\right]+E\left[U\left(V_{T}^{c}\left(\theta^{m}\right)\right) 1_{\Omega \backslash\left(A_{n} \cup A_{m}\right)}\right]\right) \\
& \quad \geq E\left[U\left(V_{T}^{c}(\theta)\right)\right]-\varepsilon
\end{aligned}
$$

and hence

$$
E\left[U\left(\frac{V_{T}^{c}\left(\theta^{n}\right)+V_{T}^{c}\left(\theta^{m}\right)}{2}\right)\right] \geq E\left[U\left(V_{T}^{c}(\theta)\right)\right]+\alpha+((\beta-1) \alpha-\varepsilon(\beta+1)) .
$$

Since $\varepsilon$ can be chosen arbitrarily small, we can assume that the last term on the right-hand side is positive, but this leads to a contradiction, since $E\left[U\left(V_{T}^{c}(\theta)\right)\right]+\alpha$ is the supremum.

5.2. Shortfall minimization. The problem of shortfall hedging is obtained choosing $\rho(X)=E\left[l\left(X^{-}\right)\right]$, where the loss function $l: \mathbb{R}^{+} \mapsto \mathbb{R}^{+}$is increasing and convex.

In this case, all the conditions in Definition 4.1 are satisfied, since $X^{-} \geq 0$ for all $X$ and hence the Fatou property (iii) always holds. The existence result then sounds as follows:

COROLlaRY 5.4. Let $X$ be a quasi-left-continuous process satisfying Assumption 2.4 and the $(\mathrm{CAF})$ condition. Then the problem

$$
\max _{\theta \in \mathcal{A}_{c}^{k}}\left[l\left(\left(V_{T}^{c}(\theta)-H\right)^{-}\right)\right]
$$

admits a solution. 
5.3. The edge of the no-arbitrage condition. As mentioned in Section 3, the compactness result of Proposition 3.2 may fail when $\gamma^{i}=k^{i}$ for some $i$, despite the market remains free of strong arbitrage opportunities. This phenomenon is best illustrated by the next counterexample.

EXAMPLE 5.5. Let $R_{t}$ be a Brownian motion started in 1 and reflected between $\frac{1}{1+\varepsilon}$ and $\frac{1}{1-\varepsilon}$, and consider a market with only one risky asset $X_{t}=R_{\frac{t}{T-t}}$.

Note that $1-\varepsilon \leq \frac{1}{X_{t}} \leq 1+\varepsilon$, and by Proposition 2.10 the asset $X$ is arbitrage free for $k \geq \varepsilon$ (since $\tilde{X}_{t} \equiv 1$ is obviously a martingale under $Q=P$ ).

Consider the stopping times $\left\{\tau_{i}\right\}_{i \geq 1}$ and $\left\{\sigma_{i}\right\}_{i \geq 0}$ defined as follows:

$$
\left\{\begin{aligned}
\sigma_{0} & =0, \\
\tau_{i+1} & =\inf \left\{s>\sigma_{i}: X_{s}=\frac{1}{1+\varepsilon}\right\}, \\
\sigma_{i} & =\inf \left\{s>\tau_{i}: X_{s}=\frac{1}{1-\varepsilon}\right\},
\end{aligned}\right.
$$

and the strategy $\theta$ defined by

$$
\theta_{t}= \begin{cases}0 & \text { for } t \in\left(\sigma_{k}, \tau_{k+1}\right], \\ \delta & \text { for } t \in\left(\tau_{k}, \sigma_{k}\right] .\end{cases}
$$

Note that by construction $\sigma_{i}, \tau_{i}<\infty$ a.e. for all $i$.

For $k=\varepsilon$, it is easy to see that $V_{t}^{c}(\theta) \in\left[c-\delta \frac{k}{1+\varepsilon}, c+\delta \frac{k}{1-\varepsilon}\right]$, and hence $\theta$ is admissible for some small $\delta>0$. Also, $|D \theta|_{\sigma_{i}}=2 \delta i$, hence $|D \theta|_{T}=\infty$ a.e.

Defining $\theta^{n}=\theta^{\sigma_{n}}$ (i.e., $\theta$ stopped at $\sigma_{n}$ ), we obtain that $\left|D \theta^{n}\right|_{T}=2 \delta n$ and hence $\left\{\left|D \theta^{n}\right|_{T}\right\}_{n}$ is not bounded in $L^{0}$. Also, all sequences of convex combinations of $\theta^{n}$ converge to $\theta$ a.s., hence there is no hope that one of them converges to a function of finite variation.

In practice, the asset $X$ in the above example allows a trivial arbitrage strategy: buy at $\frac{1}{1+\varepsilon}$; sell at $\frac{1}{1-\varepsilon}$. However, with transaction costs, this strategy remains an arbitrage depending on the cost size: for $k<\varepsilon$, it still delivers a profit, while for $k>\varepsilon$ it leads to a net loss (hence $\theta$ is not admissible). At the critical value $k=\varepsilon$, the trading gain is exactly offset by the transaction cost, and the portfolio value remains bounded though the trading strategy becomes more and more hectic as $t \rightarrow T$.

However, note that in the above example the market is not free of weak arbitrage opportunities, as there exist strategies $\theta$ such that $V_{t}^{0}(\theta) \geq 0$ a.s., and $\theta_{t} \neq 0$. In other words, agents cannot expect riskless profits with positive probability, but may well expect to build a position at no charge, thus circumventing transaction costs. 
Acknowledgments. The author thanks Maurizio Pratelli for several remarks and references, Walter Schachermayer for an illuminating discussion and an anonymous referee for suggesting several improvements. Financial support from the University of Pisa is gratefully acknowledged.

\section{REFERENCES}

[1] Cvitanić, J. (2000). Minimizing expected loss of hedging in incomplete and constrained markets. SIAM J. Control Optim. 38 1050-1066 (electronic).

[2] CVitanić, J. and KARATZAS, I. (1996). Hedging and portfolio optimization under transaction costs: a martingale approach. Math. Finance 6 133-165.

[3] Delbaen, F. (2000). Coherent risk measures on general probability spaces. Preprint, ETH Zurich.

[4] Delbaen, F. and Schachermayer, W. (1994). A general version of the fundamental theorem of asset pricing. Math. Ann. 300 463-520.

[5] Dellacherie, C. and Meyer, P. A. (1978). Probabilities and Potential. North-Holland, Amsterdam.

[6] Dellacherie, C. and Meyer, P. A. (1982). Probabilities and Potential B. North-Holland, Amsterdam.

[7] Guasoni, P. (2002). Optimal investment problems under market frictions. Ph.D. thesis, Scuola Normale Superiore, Pisa.

[8] Guasoni, P. (2002). Risk minimization under transaction costs. Finance and Stochastics 6 91-113.

[9] Jouini, E. and Kallal, H. (1995). Martingales and arbitrage in securities markets with transaction costs. J. Econom. Theory 66 178-197.

[10] Kabanov, Y. and LAST, G. (2001). Hedging under transaction costs in currency markets: a continuous-time model. Math. Finance 12 63-70.

[11] Kabanov, Y. M., RÁsonyi, M. and Stricker, C. (2001). On the closedness of sums of convex cones in $l^{0}$ and the robust no-arbitrage property. Preprint.

[12] Kabanov, Y. M. and Stricker, C. (2001). The Harrison-Pliska arbitrage pricing theorem under transaction costs. J. Math. Econom. 35 185-196.

[13] Karatzas, I., Lehoczky, J. P., Shreve, S. E. and XU, G.-L. (1991). Martingale and duality methods for utility maximization in an incomplete market. SIAM J. Control Optim. 29 702-730.

[14] Kramkov, D. O. (1996). Optional decomposition of supermartingales and hedging contingent claims in incomplete security markets. Probab. Theory Related Fields 105 459479.

[15] Kramkov, D. and Schachermayer, W. (1999). The asymptotic elasticity of utility functions and optimal investment in incomplete markets. Ann. Appl. Probab. 9 904-950.

[16] KREPS, D. M. (1981). Arbitrage and equilibrium in economies with infinitely many commodities. J. Math. Econom. 8 15-35.

[17] Merton, R. C. (1969). Lifetime portfolio selection under uncertainty: the continuous time case. Rev. Econom. Statist. 51 247-257.

[18] Schachermayer, W. (2001). The fundamental theorem of asset pricing under proportional transaction costs in finite discrete time. Preprint, TU-Wien. 
[19] SChAChermayer, W. (2001). Utility maximization in incomplete financial markets. Lecture notes, Scuola Normale Superiore, Pisa.

[20] Yan, J. A. (1980). Caractérisation d'une classe d'ensembles convexes de $L^{1}$ ou $H^{1}$. Seminar on Probability XIV. Lecture Notes in Math. 784 220-222. Springer, Berlin.

Dipartimento di Matematica

UNIVERSITÀ DI PISA

VIA BUONARROTI, 2

56127 PISA

ITALY

E-MAIL: guasoni@dm.unipi.it 